\title{
Restauration fonctionnelle du rachis : effet du niveau initial de douleur sur les performances des sujets lombalgiques chroniques
}

\author{
Isabelle Caby $\mathrm{PhD}^{1,2,3}$, N Olivier $\mathrm{PhD}^{2,3}, \mathrm{~F}$ Mendelek $\mathrm{PhD}^{4}$, R Bou Kheir $\mathrm{PhD}^{5}$, \\ J Vanvelcenaher $\mathrm{MD}^{6}$, $\mathrm{P}$ Pelayo $\mathrm{PhD}^{2,3}$
}

I Caby, N Olivier, F Mendelek, R Bou Kheir, J Vanvelcenaher, P Pelayo. Restauration fonctionnelle du rachis : effet du niveau initial de douleur sur les performances des sujets lombalgiques chroniques. Pain Res Manag 2014;19(5):e133-e138.

HISTORIQUE : La lombalgie chronique est une douleur lombaire persistante d'origine multifactorielle. Le niveau de douleur initial reste faiblement utilisé pour analyser et comparer les réponses des patients lombalgiques au programme de reconditionnement.

OBJECTIFS : Apprécier et évaluer les réponses des sujets lombalgiques chroniques très douloureux à une prise en charge dynamique et intensive. MÉTHODOLOGIE : 144 sujets atteints de lombalgie chronique ont été inclus dans un programme de restauration fonctionnelle du rachis de 5 semaines. Les sujets ont été classés en deux groupes de niveau de douleur : un groupe atteint de douleur sévère $(n=28)$ et un groupe atteint de douleur légère à modérée $(n=106)$. L'ensemble des sujets ont bénéficié d'une prise en charge identique comprenant principalement de la kinésithérapie, de l'ergothérapie, du reconditionnement musculaire et cardio-vasculaire ainsi qu'un suivi psychologique. Les paramètres physiques (flexibilité, force musculaire) et psychologiques (qualité de vie) ont été mesurés avant (T0) et après le programme (T5sem)

RÉSULTATS : L'ensemble des performances physiques et fonctionnelles des sujets très douloureux sont moins bonnes et le retentissement de la lombalgie sur la qualité de vie, pour ces mêmes sujets, est majoré à T0. Toutes les différences significatives constatées à T0 entre les deux groupes s'effacent à T5sem.

CONCLUSIONS : Les sujets lombalgiques chroniques très douloureux répondent favorablement au programme dynamique et intensif. L'intensité douloureuse de la lombalgie n'aurait pas d'effet sur les réponses au programme. La restauration fonctionnelle du rachis apporterait aux sujets la possibilité de mieux gérer leur douleur quel que soit son niveau.

Key Words: Douleur; Rachis lombaire; Restauration fonctionnelle; Troubles musculo-squelettiques

T es troubles musculo-squelettiques figurent parmi les principales Lcauses d'arrêt de travail. Le mal de dos, appelé aussi lombalgie, y tient une place majeure et s'est inscrit, au XXème siècle, au rang des maladies les plus fréquentes et les plus invalidantes (1). De nombreux pays industrialisés ont eu à faire face à ce véritable problème de santé public et continuent d'y remédier au XXIème siècle.

Les maux de dos constituent la principale cause d'incapacité à travailler chez les moins de 45 ans et la troisième chez les 45 ans et plus (2). Il s'agit d'un problème de santé plus coûteux que le sida, le cancer ou les maladies du cœur. En fait, les lombalgies constituent un problème tellement important que l'Organisation Mondiale de la Santé (OMS) a fait des années 2000 à 2010 « la décennie des os et des articulations " (3). Cette décennie amène une véritable prise de conscience du poids considérable des conséquences humaines et
Functional restoration of the spine: Effect of initial pain level on the performance of subjects with chronic low back pain

BACKGROUND: Chronic low back pain is a persistent lumbar pain of multifactorial origin. The initial pain level remains poorly used to analyze and compare responses in low back pain patients in a reconditioning program. OBJECTIVE: To assess and evaluate the responses of subjects with very painful chronic low back pain in a dynamic and intensive care program. METHODS: A total of 134 patients with chronic low back pain were included in a spine functional restoration program for five weeks. The subjects were classified into two groups by level of pain: a group experiencing severe pain $(n=28)$ and a group experiencing mild to moderate pain $(\mathrm{n}=106)$. All subjects received identical support consisting primarily of physiotherapy, occupational therapy, cardiovascular and muscular reconditioning as well as psychological counselling. The physical parameters (flexibility, muscular strength) and psychological (quality of life) were measured before (TO) and after the program (T5sem).

RESULTS: All physical and functional performances of the subjects with severe pain were lower and the impact of back pain on quality of life for these subjects was increased. All significant differences at TO between the two groups were no longer present at T5sem.

DISCUSSION: Muscular atrophy is more important in subjects with severe pain at TO. The intense pain would cause kinesiophobia and central inhibition in chronic low back pain. The analgesic effects of the spine functional restoration program allowed subjects to obtain similar physical, functional and psychological performances at the end of the five weeks of support.

CONCLUSIONS: Patients with very painful chronic low back pain respond favourably to the dynamic and intensive program. The intensity of low back pain had no effect on responses to the program. The spine functional restoration program enables patients to better manage their pain, whatever its level.

${ }^{1}$ Department of Sports Sciences, Artois University, Liévin; ${ }^{2}$ Université Lille-Nord de France; ${ }^{3}$ UDSL, EA4488, Ronchin, France; ${ }^{4}$ Sacré-Cour

Hospital, Physiotherapy Service, Baabda; ${ }^{5}$ Lebanese University, Fanar, Lebanon; ${ }^{6}$ Department of Physical Medicine and Readaptation,

Functional Re-education and Rehabilitation center, "L'Espoir", Hellemmes, France

Correspondance: Dr Isabelle Caby, Faculté des Sports et de l'EP de Liévin, chemin du marquage, 62800 Liévin, France.

Telephone 00-33-3-21-45-88-44 / 00-33-3-21-45-85-06, fax 00-33-3-21-45-85-01, e-mail i.caby@wanadoo.fr 
limitations d'activités $(6,7)$. Le syndrome de déconditionnement se traduit par une hypo-extensibilité musculo-tendineuse notamment au niveau des ischios-jambiers. Le manque d'activité quotidienne engendré par les souffrances lombaires entraîne une baisse de l'aptitude aérobie et une atrophie des différentes fibres musculaires (8) ; une diminution de la musculature des parois antérieures et postérieures du tronc est constatée (9). A ce déconditionnement physique s'ajoute une désocialisation progressive. En plus de l'arrêt de travail provoquant une perte d'identité professionnelle, la grande majorité des sujets lombalgiques évoque également un arrêt des activités de loisirs provoquant une perte de contact avec l'entourage. Tout cela ayant pour conséquence une diminution de leur qualité de vie (10).

Pour faire face à ce syndrome, le programme de Restauration Fonctionnelle du Rachis (RFR) a la particularité d'assurer une prise charge globale et unifiée, en temps et en lieu, du sujet lombalgique chronique (11). L'efficacité du programme de restauration fonctionnelle du rachis est appréciée à partir des performances physiques et des tests psychosociaux mais aussi et surtout à partir de la reprise d'une activité professionnelle (12).

Le déconditionnement physique est la cible première du traitement, associée à la modification des facteurs psychosociaux afin de corriger les croyances et le comportement du sujet. Le stress, l'anxiété et la dépression sont trois états psychologiques souvent connus chez les sujets atteints de lombalgie chronique. Non seulement toute douleur chronique est anxiogène, mais en plus, l'absence d'étiologie du symptôme douleur est souvent considérée par le lombalgique chronique comme un facteur angoissant supplémentaire. La littérature est assez ambiguë sur la relation entre la douleur et la dépression. Certains pensent que la « dépression aggrave la douleur » ou encore que « la douleur chronique déclenche une dépression ». Pourtant l'étude menée par Genêt et al. en 2006 n'a trouvé aucune corrélation entre intensité douloureuse et dépression. Selon eux, ces facteurs seraient indépendants (13).

Face à la douleur, le sujet lombalgique chronique développe des comportements d'évitement et d'appréhension (peur de se faire mal, de la douleur, de l'activité). Des appréhensions pour les activités physiques et les activités professionnelles qui, si elles persistent, entrainent une diminution des activités, une baisse de motivation et amènent à un syndrome de déconditionnement. Ce syndrome de déconditionnement est complexe, pluriel et surtout multifactoriel. Il nécessite de proposer au sujet lombalgique chronique une prise en charge adaptée capable de répondre aux problèmes physiques, fonctionnels, psychologiques, sociaux et professionnels de la pathologie.

Le traitement prescrit doit viser l'antalgie, la récupération des déficiences physiques, la prévention des récidives et l'information du patient. Une nouvelle génération de médecins considère la douleur comme le cinquième signe vital après la pulsation, la tension artérielle, la respiration et la température (14). Le niveau de douleur est devenu un élément central des programmes de prise en charge des sujets lombalgiques (15). L'échelle visuelle analogique (EVA) constitue aujourd'hui un outil fiable et reproductible pour mesurer le niveau de douleur (16). L'échelle est étalonnée de 0 à 100 millimètres. Elle est limitée par deux extrémités : l'extrémité de gauche correspond à une " absence de douleur », tandis que l'extrémité de droite correspond à une « douleur maximale imaginable ». Le sujet doit indiquer sur l'échelle l'intensité de sa douleur au cours des trois derniers jours. Le score EVA correspond à la distance mesurée en millimètres $(\mathrm{mm})$. A partir de cette échelle de mesure, Huskisson a pu identifier trois niveaux de douleur : léger (score $<30 \mathrm{~mm}$ ), moyen (score compris entre 31 et $69 \mathrm{~mm}$ ), et sévère (score $>70 \mathrm{~mm}$ ). L'EVA est devenue un des outils les plus utilisés par la communauté scientifique pour mesurer l'intensité de la douleur. Bien que l'utilisation de cette échelle visuelle soit répandue et reconnue, peu d'études s'intéressent à analyser les réponses en fonction du niveau initial de douleur (16). Des travaux récents ont mis en évidence des performances minorées chez les sujets lombalgiques atteints de douleur sévère (17).
Dans notre étude, nous avons cherché à analyser les réponses des patients lombalgiques au programme de restauration fonctionnelle du rachis en fonction de leur niveau initial de douleur. Ceci dans l'objectif d'étudier l'effet de l'intensité douloureuse sur les réponses au programme.

\section{MÉTHODOLOGIE}

\section{Population}

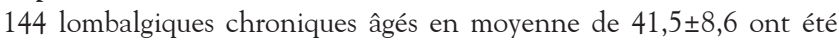
inclus dans un programme de restauration fonctionnel du rachis après une évaluation physique d'inclusion. Toutes les personnes sont volontaires et adressées par leur médecin traitant.

L'ensemble des sujets ont participé à une Évaluation Physique d'Inclusion (EPI) pour identifier la présence d'un syndrome de déconditionnement, défini par Mayer et al (18). Cette E.P.I comprend une évaluation physique (test de flexibilité, tests isocinétiques mesurant la force et l'endurance musculaire des fléchisseurs et extenseurs du tronc, test fonctionnel de soulever de charge), une évaluation psychométrique et psychiatrique (analyse des indices de dépression, d'anxiété, de motivation et de participation, analyse de la personnalité du sujet et de l'influence des circonstances extérieures : histoire du patient, entourage, vécu des expériences antérieures) et une évaluation socioprofessionnelle où l'assistant(e) social(e) recueille des informations sur les domaines professionnels, familiaux et financiers.

A l'issue de cette EPI, le médecin rééducateur pouvait apprécier la présence effective d'un syndrome de déconditionnement chez le sujet lombalgique chronique à inclure, ainsi qu'une personnalité compatible avec une reprise d'activité physique et/ou professionnelle.

Les critères d'inclusion étaient : âge entre 18 et 65 ans, présence d'une lombalgie chronique de plus de trois mois, présence d'un syndrome de déconditionnement et situation d'impasse thérapeutique.

Les critères d'exclusion étaient : troubles psychologiques (dépression, anxiété, troubles psychiatriques), causes organiques habituellement reconnues (discopathie évolutive aiguë ou subaiguë, pathologie inflammatoire, tumorale ou infectieuse, atteinte neurologique, problème viscéral, pathologie cardiaque), bénéfices secondaires (financiers), dépendance sévère au tabac, à l'alcool.

Après l'évaluation du niveau de douleur initial (EVA à TO), les sujets ont été classés en deux groupes : un groupe avec un niveau de douleur initial (DI) "faible à modérée" pour lequel les scores de douleur (EVA) sont compris entre 0 et 69 (DI $<70 \mathrm{~mm}$ ); un groupe avec un niveau de douleur initial (DI) "sévère" dont les scores de l'échelle visuelle analogique sont compris entre 70 et $100 \mathrm{~mm}(\mathrm{DI} \geq 70)$.

\section{Intervention}

Le programme de restauration fonctionnel du rachis repose sur une prise en charge multidisciplinaire de la douleur, avec l'intervention de professionnels variés : médecins, ergothérapeutes, kinésithérapeutes, psychologues, psychiatres, professeur d'Education Physique, assistantes sociales. L'objectif principal du programme est de restaurer le mouvement et les possibilités fonctionnelles du sujet plutôt que de supprimer la douleur. Les patients s'entraînaient 7 heures par jour, 5 jours par semaine, pendant 5 semaines, soit un total de 175 heures d'entraînement. Le programme comprenait un reconditionnement musculaire du tronc et des muscles des membres, des exercices cardiovasculaires sur rameurs, ergocycles à membres supérieurs et inférieurs, des étirements, de la proprioception, de l'ergothérapie, de la relaxation, de la balnéothérapie et des activités physiques et sportives. Le médecin supervisait les exercices effectués et ajustait l'intensité des exercices à chaque patient lombalgique chronique. Pendant la première semaine, les patients réalisaient un travail de flexibilité et des exercices cardio-respiratoires. Pendant la deuxième semaine, les patients commençaient un travail de force musculaire. Pendant la troisième semaine, le renforcement musculaire été augmenté avec des exercices d'endurance. Les patients effectuaient des soulevés de charges ainsi que des exercices de proprioception et de la coordination. Au cours des quatrièmes et cinquièmes semaines, l'intensité des exercices de renforcement augmentait progressivement. 
L'entraînement cardio-vasculaire (marche, course, pédalage) était adapté à chaque patient et individualisé. Une fois par semaine les sujets lombalgiques bénéficiaient d'une consultation avec le psychologue si nécessaire. Une prise en charge psychologique et éducative était également assurée chaque semaine pour l'ensemble des sujets par l'intermédiaire d'enseignements anatomo-physiologiques de base sur le rachis, d'informations sur les bénéfices du mouvement et sur la gestion de la douleur (distinction entre la douleur pathologique et non pathologique liée à l'entraînement). Des consultations avec l'assistante sociale étaient proposaient chaque semaine pour préparer le retour du patient au travail et les conditions de reprise. Une synthèse était organisée chaque semaine pour faire le bilan des progrès du patient.

Une journée type se déroulait le matin avec un échauffement sur rameur ou ergocycle à membres supérieurs, un entraînement musculaire isocinétique, un entraînement musculaire analytique des membres inférieurs et supérieurs sur machines à charge, de la préparation physique générale ou une consultation avec l'assistant social ou le psychologue ; l'après-midi : un entraînement fonctionnel en ergothérapie, un entraînement musculaire isocinétique, un entraînement musculaire analytique des muscles du tronc sur machines à charge, de l'activité physique et sportive, des étirements, de la balnéothérapie et/ou de la relaxation.

\section{Protocole expérimental}

L'étude s'est effectuée en centre de rééducation fonctionnelle. De façon à observer les adaptations et réponses au programme, deux évaluations ont été programmées. La première (TO) a été réalisée le premier jour de l'hospitalisation avant le reconditionnement à l'effort, la seconde $(\mathrm{T} 5 \mathrm{sem})$ se situe à la fin des cinq semaines de rééducation. Pour limiter au maximum les variations biologiques, les tests ont toujours été effectués aux mêmes heures et dans des conditions identiques. L'évaluation initiale a été précédée d'exercices de familiarisation afin que les postures soient correctement réalisées. Les sujets des deux groupes de niveau de douleur initial ont vécu sans distinction, le même programme de restauration fonctionnelle du rachis.

\section{Paramètres mesurés}

\section{Données démographiques et cliniques:}

Pour chaque patient le médecin rééducateur du centre recueillait les données concernant l'âge, le poids, la taille, la durée de la lombalgie, la durée de l'arrêt de travail et la consommation de tabac (tableau 1). L'entretien clinique permettait également de connaître la consommation d'antalgiques et la pratique d'activité physique et sportive (bricolage, marche, activités sportives, professionnelles et de loisirs).

La qualité de vie:

Elle est évaluée avec l'autoquestionnaire de DALLAS (19). Ce questionnaire est une échelle spécifique, validée en français, évaluant le retentissement de la douleur lombaire dans quatre domaines : les activités de la vie quotidienne, les activités professionnelles et de loisirs, le rapport anxiété-dépression et la sociabilité. Les résultats s'expriment en pourcentage allant de $0 \%$ (absence du retentissement) à $100 \%$ (gêne maximale).

Un autre questionnaire a été soumis aux patients de façon à connaître leur consommation de médicaments (antalgiques, somnifères) et le temps consacré à une activité physique (bricolage, marche, activités sportives, professionnelles et de loisirs).

\section{La douleur:}

Les patients ont évalué leur douleur à partir d'une échelle visuelle analogique (EVA) étalonnée de 0 à 100 millimètres. Elle est limitée par deux extrémités : l'extrémité de gauche correspond à une « absence de douleur », tandis que l'extrémité de droite correspond à une « douleur maximale imaginable ». Le sujet doit indiquer sur l'échelle l'intensité de sa douleur au cours des trois derniers jours. Le score EVA correspond à la distance mesurée en millimètres. Dans ce mode d'évaluation, le 0 correspond à une absence de douleur et le $100 \mathrm{au}$ niveau le plus intense (16).

\section{La flexibilité:}

La souplesse du plan postérieur a été testée à l'aide de la distance doigts sol (20) et de la distance doigts-pieds (DDP). La distance doigts-sol, DDS,
$(21,22)$ mesure, dans un plan sagittal, la distance séparant l'extrémité du médius du niveau de la plante des pieds. Le sujet est sur un podium et réalise après deux essais d'échauffement, une flexion maximale du tronc sans fléchir les genoux. La distance doigts-pieds, DDP (23), se réalise en position assise au sol. Ce test de flexibilité permet d'éviter l'appréhension de flexion du tronc en limitant le travail freinateur des muscles postérieurs. De la même manière que dans le test précédent, le sujet effectue, jambes tendues, une flexion maximale du tronc. Le résultat $(\mathrm{en} \mathrm{cm}$ ) indique la distance entre l'extrémité du médius et la plante des pieds.

La capacité musculaire:

Lévaluation musculaire des fléchisseurs et extenseurs du tronc a été réalisée sur un appareil isocinétique régulièrement calibré (Cybex Norm $\left.{ }^{\circledR}\right)$. Ce test est précédé d'un échauffement sur rameur d'une durée de 10 minutes. Les personnes sont en position debout, les membres inférieurs sont stabilisés par des contre-appuis, genoux à $20^{\circ}$ de flexion. Le mouvement testé est la flexion/extension autour d'un axe horizontal passant par l'interligne L5-S1. L'amplitude du mouvement est de $70^{\circ}$. L'évaluation est réalisé à trois vitesses $(30 \%$ s, $90 \%, 120 \%$ s), sur un mode concentrique, avec respectivement pour chacune d'entre-elles 3 , 5 et 20 répétitions, deux minutes de repos entre chaque série. Les résultats chiffrés concernent le pic de couple à $30 \%$, la puissance à $90 \%$ et le travail total à $120 \%$ s. Pour l'ensemble de ces valeurs, un calcul du ratio fléchisseurs sur extenseurs a été effectué (F/E).

L'endurance musculaire était évaluée à l'aide du test de Sorensen (24). Le sujet est placé en décubitus ventral sur une chaise romaine, le tronc suspendu dans le vide avec un contre-appui au niveau des tendons d'Achille. Après un échauffement, le test consiste à maintenir le plus longtemps possible le tronc à l'horizontale, les bras croisés sur la poitrine. L'épreuve s'arrête lorsque la position n'est plus maintenue et/ ou lorsqu'une douleur lombaire ou pelvienne apparaît. La durée de maintien de la position est mesurée à l'aide d'un chronomètre et le résultat est indiqué en secondes.

Le soulever de charge:

Le test de soulever de charge (25) est une adaptation du test américain PILE (Progressive Isoinertial Lifting Evaluation) (26). Ce test dynamique évalue l'endurance à l'effort, la capacité physique et la performance fonctionnelle. Le sujet doit soulever une caisse du sol et la poser sur un plateau situé à $75 \mathrm{~cm}$ de hauteur et la remettre au sol. Le sujet doit réaliser 4 levers de caisse (ou 8 mouvements) en 20 secondes. Le test s'arrête soit à la demande du sujet, soit parce que le temps imparti pour effectuer le cycle des 4 levers est dépassé. La charge initiale est de 2,5 kg pour les femmes, $5 \mathrm{~kg}$ pour les hommes, incrémentée de $2,5 \mathrm{~kg}$ et de $5 \mathrm{~kg}$ respectivement.

\section{Analyse statistique}

Les résultats sont présentés sous forme de moyenne et d'écart type. L'analyse statistique est réalisée au moyen du logiciel Sigma STAT version 2.03. Afin de comparer les différentes données entre les deux groupes, un "t" test a été utilisé. Pour affiner l'interprétation des paramètres étudiés, le seuil de significativité est fixé à 5\%.

\section{RESULTATS}

144 sujets ont été inclus dans le programme RFR de janvier 2002 à décembre 2006. Des 144 sujets, 10 sujets ont été sortis de l'étude pour cause d'évaluation incomplète. Il n'y a eu aucun abandon pour le programme proposé. La comparaison des deux groupes de douleur repose sur un effectif global de 134 sujets : 106 sujets pour le groupe de douleur initiale faible à modérée $(\mathrm{DI}<70 \mathrm{~mm})$ et 28 sujets pour le groupe de douleur initiale sévère $(\mathrm{DI} \geq 70)$. Les 134 sujets sont âgés en moyenne de $41,5 \pm 8,6$ ans. Les sujets du groupe DI sévère sont légèrement plus âgés que le groupe DI légère à modérée $(\mathrm{p}<0,05): 44,7 \pm 8,3$ vs $40,7 \pm 8,2$ respectivement. En dehors de l'âge, les caractéristiques des sujets des deux groupes de douleur (tableau 1) sont identiques.

L'Indice de Masse Corporelle moyen est de $24,9 \pm 4,1 \mathrm{~kg} / \mathrm{m}^{2} .46 \%$ des sujets lombalgiques chroniques de notre étude sont en surpoids $($ IMC $>25)$ avec une proportion plus importante pour les pour les hommes que pour les femmes. Les patients signalaient des lombalgies depuis $74 \pm 64$ mois. La lombalgie chronique est responsable des arrêts 
TABLEAU 1

Caractéristiques des deux groupes de douleur initiale

\begin{tabular}{|c|c|c|c|c|}
\hline & $\mathrm{DI}<70(\mathrm{n}=106)$ & $\mathrm{DI} \geq 70(\mathrm{n}=28)$ & $\mathbf{P}$ & Moyenne $\pm \mathrm{ET}$ \\
\hline Age, années & $40,7 \pm 8,3$ & $44,7 \pm 8,2$ & $P<0.05$ & $41,5 \pm 8,6$ \\
\hline Taille, $m$ & $1,72 \pm 0,1$ & $1,70 \pm 0,1$ & ns & $1,71 \pm 0,1$ \\
\hline Durée moyenne des arrêts de travail (sem) & $32 \pm 37$ & $37 \pm 46$ & ns & $39 \pm 39$ \\
\hline Durée moyenne de la lombalgie (mois) & $75 \pm 64$ & $71 \pm 70$ & ns & $74 \pm 64$ \\
\hline Consommation de tabac (\%) & 32 & 34 & ns & 33 \\
\hline
\end{tabular}

Valeurs présentées en moyenne et écart type (ET) ou pourcentage \%. DI Douleur initiale ; IMC Indice de masse corporelle ; $m$ Mètre ; sem Semaine. Significativité $:{ }^{*} p<0.05 ; n s$ Non significatif

TABLEAU 2

Effets du niveau initial de douleur sur les paramètres physiques, fonctionnels et psychologiques

\begin{tabular}{|c|c|c|c|c|c|c|}
\hline & \multicolumn{3}{|c|}{ T0 } & \multicolumn{3}{|c|}{ T5sem } \\
\hline & DI $<70$ & $\mathrm{DI} \geq 70$ & $\mathbf{P}$ & DI $<70$ & $\mathrm{DI} \geq 70$ & $\mathbf{P}$ \\
\hline Paramètres & \multicolumn{6}{|c|}{ Moyenne \pm ET Significativité } \\
\hline DDS, cm & $12 \pm 13$ & $18 \pm 16$ & * & $-7 \pm 7$ & $-5 \pm 8$ & ns \\
\hline DDP, $\mathrm{cm}$ & $6 \pm 11$ & $10 \pm 13$ & ns & $-8 \pm 8$ & $-7 \pm 9$ & ns \\
\hline EDI, degrés & $91 \pm 24$ & $82 \pm 21$ & ns & $118 \pm 15$ & $116 \pm 16$ & ns \\
\hline EVA, mm & $42 \pm 12$ & $76 \pm 14$ & *** & $25 \pm 19$ & $38 \pm 27$ & * \\
\hline TSC, \% PdC, EPI & $26 \pm 12$ & $22 \pm 10$ & ns & $46 \pm 14$ & $39 \pm 16$ & ns \\
\hline Sorensen, sec & $65 \pm 47$ & $37 \pm 33$ & ** & $110 \pm 48$ & $102 \pm 45$ & ns \\
\hline Ratio F/E $30^{\circ} \mathrm{sec}, \mathrm{MFM}, \% \mathrm{PdC}$ & $1,08 \pm 0,27$ & $1,09 \pm 0,29$ & ns & $0,88 \pm 0,20$ & $0,78 \pm 0,14$ & * \\
\hline Ratio F/E $120^{\circ} \mathrm{sec}, \mathrm{P}, \% \mathrm{PdC}$ & $1,45 \pm 0,85$ & $1,52 \pm 0,73$ & ns & $1,06 \pm 0,36$ & $1,04 \pm 0,53$ & ns \\
\hline $\begin{array}{l}\text { Extenseurs du tronc, Force maximale } 30^{\circ} \mathrm{sec} \text {, } \\
\text { Pic de Couple, \% PdC }\end{array}$ & $228 \pm 81$ & $205 \pm 72$ & ns & $310 \pm 93$ & $303 \pm 72$ & ns \\
\hline $\begin{array}{l}\text { Extenseurs du tronc, Force endurance } 120^{\circ} \mathrm{sec} \text {, } \\
\text { Travail Total, \% PdC }\end{array}$ & $114 \pm 64$ & $75 \pm 47$ & ** & $215 \pm 70$ & $199 \pm 63$ & ns \\
\hline $\begin{array}{l}\text { Extenseurs du tronc, Force vitesse } 90^{\circ} \mathrm{sec} \text {, } \\
\mathrm{P}, \% \mathrm{PdC}\end{array}$ & $136 \pm 69$ & $95 \pm 54$ & $* * *$ & $240 \pm 81$ & $226 \pm 70$ & ns \\
\hline Dallas AQ, \% & $66 \pm 17$ & $77 \pm 13$ & * & $37 \pm 26$ & $49 \pm 24$ & ns \\
\hline Dallas AP, \% & $62 \pm 18$ & $77 \pm 16$ & * & $37 \pm 26$ & $39 \pm 25$ & ns \\
\hline Dallas AD, \% & $46 \pm 25$ & $64 \pm 22$ & * & $21 \pm 21$ & $29 \pm 28$ & ns \\
\hline Dallas S, \% & $33 \pm 20$ & $33 \pm 28$ & ns & $24 \pm 23$ & $24 \pm 20$ & ns \\
\hline
\end{tabular}

TO Évaluation à l'entrée ; T5sem Évaluation à la fin des 5 semaines de programme RFR ; DI < 70 Douleur initiale inférieure à 70 millimètres ; DI $\geq 70$ Douleur initiale supérieure ou égale à 70 millimètres ; DDS Distance doigts-sol ; DDP Distance doigts-pieds ; EDI Évaluation de l'inclinométrie ; EVA Échelle visuelle analogique ; TSC Test de soulever de charge ; ratio F/E Ratio fléchisseurs sur extenseurs ; cm Centimètre ; mm Millimètre ; \% PdC Pourcentage de poids de corps ; sec Seconde ; MFM Moment de force maximale ; P Puissance moyenne ; AQ Activités de la vie quotidienne ; AP Activités de la vie professionnels et loisirs ; AD Anxiétédépression; $S$ Sociabilité. Les cases grisées correspondent à l'absence d'évaluation. Différence Douleur initiale légère à modérée - Douleur initiale sévère à T0 et T5sem : * $p<0,05 ;{ }^{* *} p<0,01 ;{ }^{* * *} p<0,001$

de travail pour près de deux tiers des patients avec une durée moyenne de $39 \pm 39$ semaines. Un tiers des patients a subi une chirurgie du rachis (31\% pour les sujets du groupe douleur initiale faible à modérée et $15 \%$ pour les sujets du groupe DI sévère) et un tiers est fumeur avec une consommation moyenne de tabac de $6 \pm 11$ cigarettes/jour. Seul un quart des patients lombalgiques pratiquent une activité physique régulière ( 2 fois ou plus par semaine). Professionnellement, les patients sont majoritairement issus des CSP « employés » et «professions intermédiaires » pour $38 \%$ et $26 \%$ d'entre eux respectivement.

Les résultats des sujets très douloureux $(\mathrm{DI} \geq 70 \mathrm{~mm})$ à $\mathrm{TO}$ sont significativement différents et moins bons pour la flexibilité, pour les performances d'endurance musculaire isométrique $(p<0,01)$ et anisométrique $(\mathrm{p}<0,01)$, de force vitesse isocinétique $(\mathrm{p}<0,001)$ et pour l'ensemble des paramètres de qualité de vie à l'exception de la sociabilité (Tableau 2). D'une manière générale, l'ensemble des performances physiques et fonctionnelles des sujets très douloureux sont plus faibles et le retentissement de la lombalgie sur la qualité de vie, pour ces mêmes sujets, est majoré. Toutes les différences significatives constatées à T0 s'effacent à T5sem. Une nouvelle différence significative apparaît pour le ratio "fléchisseurs/extenseurs " en faveur du groupe très douloureux. Le niveau de douleur a significativement diminué pour les deux groupes passant d'une douleur moyenne à une douleur légère pour le groupe dont la douleur initiale était inférieure à 70 (DI $<70 \mathrm{~mm})$ et passant d'une douleur sévère à une douleur moyenne pour les sujets initialement très douloureux (DI $\geq 70 \mathrm{~mm}$ ). Les sujets initialement très douloureux conservent un niveau de douleur significativement plus important à T5sem bien que minoré.

Les pourcentages d'amélioration entre T0 et T5sem (figure 1) du groupe très douloureux présente des améliorations significativement meilleures pour l'endurance musculaire isométrique (Sorensen) et anisométrique (travail total à $120 \%$ s) des extenseurs du tronc. Les ratios $\mathrm{F} / \mathrm{E}$ à vitesse lente et rapide sont également mieux améliorés pour ce même groupe $(\mathrm{p}<0,05)$. D'une manière générale les sujets du groupe DI sévère répondent très bien au programme de restauration fonctionnelle du rachis, aussi bien, si ce n'est mieux pour certains paramètres, que les sujets du groupe DI légère à modérée.

\section{DISCUSSION}

Les caractéristiques des sujets lombalgiques affichent un âge significativement plus élevé pour les sujets ayant un niveau de douleur sévère. Les données de la littérature n'ont pourtant pas trouvé de corrélation entre l'intensité douloureuse et l'âge (13). L'âge est reconnu comme 


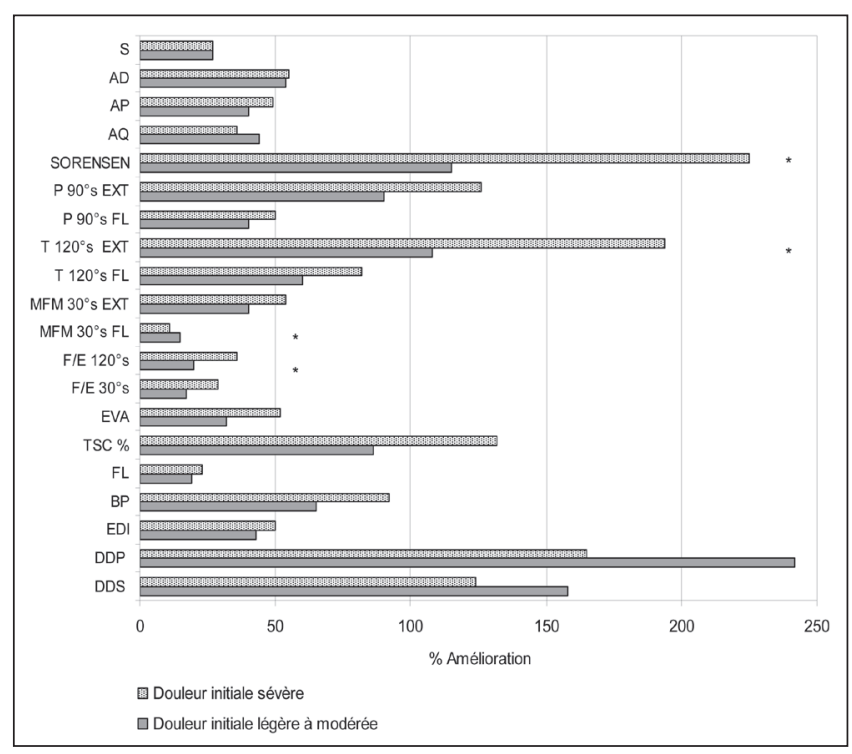

Figure 1) DDS Distance doigts-sol ; DDP Distance doigts-pieds ; EDI Évaluation de l'inclinométrie ; BP Bascule pelvienne ; FL Flexion lombaire ; TSC Test de soulever de charge ; EVA Échelle visuelle analogique ; ratio F/E Ratio fléchisseurs sur extenseurs ; MFM Moment de force maximale; T Travail Total ; P Puissance moyenne ; FL Fléchisseurs ; EXT Extenseurs ; $30^{\circ}$ s Vitesse de 30 degrés par seconde; $120^{\circ}$ s Vitesse de 120 degrés par seconde; $90^{\circ} \mathrm{s}$ Vitesse de 90 degrés par seconde ; AQ Activités de la vie quotidienne; AP Activités de la vie professionnels et loisirs; $\mathrm{AD}$ Anxiété-dépression; S Sociabilité. Différence "Douleur initiale légère à modérée " - "Douleur initiale sévère " : * $p<0,05$; ** $p<0,01$; $* * * p<0,001$

facteur de chronicisation mais pas comme un facteur aggravant la douleur (27). Le poids et l'Indice de Masse Corporelle des sujets des deux groupes sont identiques et confirment l'absence de relation entre la surcharge pondérale et l'aggravation de la douleur (28). Même si l'excès de poids est une caractéristique reconnue dans la population lombalgique (29), ce dernier n'est pourtant pas reconnu comme un facteur de risque de la lombalgie chronique $(30,31)$. La proportion plus importante de surcharge pondérale chez les sujets de sexe masculin est conforme aux données de la littérature. La proportion de fumeur est identique à celle d'études précédentes (32). Certains auteurs ont d'ailleurs trouvé une association entre le tabagisme et la lombalgie chronique (33).

Le niveau de déconditionnement physique, fonctionnel et psychologique constaté chez les sujets très douloureux à T0 est significativement plus important pour la moitié des paramètres évalués. L'atrophie des muscles du tronc pourrait être une des origines de la douleur lombaire chronique (34) et serait plus accentuée chez les sujets très douloureux. Des récepteurs nociceptifs sensibles, entre autres, aux stimuli de pression, de cisaillement et d'étirement ont été localisés au niveau musculaire. L'atrophie musculaire aurait un effet sur la douleur et inversement. Chez les sujets lombalgiques, la dramatisation de la douleur serait responsable d'appréhensions (peur du mouvement, peur de se faire mal) qui entraîneraient des conduites d'évitement (35). Mais les relations entre intensité douloureuse et comportements d'appréhension et d'évitement restent encore controversées (36). Le comportement d'évitement des sujets très douloureux pourrait expliquer les résultats, nettement minorés à $\mathrm{TO}$, d'endurance musculaire isométrique comparés aux sujets moins douloureux : $37 \pm 33$ contre $65 \pm 47$ secondes respectivement. D'autre part, les contraintes physiologiques engendrées par l'effort statique mis en jeu lors du test de Sorensen pourraient être moins bien supportées par le groupe très douloureux entraînant alors des inhibitions centrales. La diminution du flux sanguin local (37), l'évacuation difficile des catabolites (38), l'augmentation de la fréquence cardiaque, de la pression artérielle (39) et de la pression intrathoracique pourraient accentuer les influx nociceptifs. Ces influx nociceptifs renvoient ensuite à des stratégies de coping différentes (40) s'exprimant par une relation négative entre les performances et la douleur anticipée puis ressentie (41).

Les deux groupes de sujets obtiennent des résultats similaires, à T0, au test de soulever de charges. Ces résultats confirment les données de la littérature où l'intensité douloureuse n'est pas corrélée avec l'incapacité fonctionnelle (13). Les sujets très douloureux ont une qualité de vie minorée à T0, comparée aux sujets moins douloureux notamment pour les activités quotidiennes, les activités professionnelles et de loisirs et l'anxiété-dépression. L'intensité douloureuse conduirait le sujet lombalgique à mettre en place des conduites d'évitement pour les activités professionnelles. Lorsque le sujet lombalgique est persuadé que l'activité professionnelle majore la douleur, alors il met en place un comportement d'évitement, la reprise de l'activité professionnelle devient délicate et l'arrêt de travail se prolonge. Nous constatons dans notre étude, une durée de l'arrêt de travail plus importante mais non significative pour le groupe très douloureux. Pour nos sujets, l'étude des corrélations entre ces deux critères n'est pas concluante $(r=0,21)$. Aussi, nous ne pouvons pas confirmer la relation entre intensité douloureuse et durée de l'arrêt de travail.

Les résultats de notre étude affichent également un facteur anxiété significativement plus affecté lorsque la douleur est sévère, mais l'analyse des corrélations est négative. Pour certains auteurs, l'intensité douloureuse et la dépression sont considérées comme des facteurs indépendants (40), alors que, pour d'autres, ils seraient dépendants (3), laissant le débat ouvert. À l'issue du programme (T5sem), les résultats physiques, fonctionnels et psychologiques des sujets très douloureux sont identiques à ceux des sujets moins douloureux, et témoignent des effets positifs, bénéfiques et antalgiques du traitement dynamique sur la douleur. Les sujets très douloureux répondent favorablement au programme RFR, et plus favorablement que le groupe moins douloureux pour l'endurance musculaire isométrique et anisométrique des extenseurs du tronc.

En conclusion, la prise en charge multidisciplinaire, dynamique et intensive de la lombalgie chronique permettrait de modifier le comportement des sujets lombalgiques face à la douleur. Les sujets très douloureux répondent favorablement au programme RFR, aussi bien si ce n'est mieux pour certains paramètres, que les sujets moins douloureux. L'intensité douloureuse de la lombalgie n'aurait pas d'effet sur les réponses au programme.

REMERCIEMENTS : Les auteurs n'ont reçu aucune subvention ni participation financière pour cette étude. Aucun bénéfice de quelque sorte que ce soit n'a été perçu ou ne sera perçus pour cette étude. Cette étude a été menée au Centre de rééducation et de réadaptation fonctionnelles spécialisées, "L'Espoir", 25, pavé du moulin, 59260, Hellemmes, France.

\section{REFERENCES}

1. Hoy D, Brooks P, Blyth Fet al. The epidemiology of low back pain. Best Pract Res Clin Rheumatol 2010;24:76981.

2. Marty J. L'impact socio-économique des lombalgies. Gazette médicale de France 1991;98:43-6.

3. Brongel L, Lorkowski J, Hladki W, Trybus M. [Bone and joint decade - "mile step" in diagnostics and treatment of movement system diseases?]. Przegl Lek 2006;63(Suppl 5):9-13.

4. Duquesnoy B, Defontaine MC, Grardel B, et al. Définition de la lombalgie chronique. Rev Rhum 1994;61(4bis):9S-10S.

5. Merskey E, Bogduk E. Classification of Chronic Pain, 2nd edn. Washington, DC: IASP Press, 1994:209-14.

6. Mayer TG, Gatchel RJ. Functional restoration for spinal disorders: The sports medicine approach. Philadelphia: Lea and Febiger, 1988.

7. Verbunt JA, Seelen HA, Vlaeyen JW, et al. Disuse and deconditioning in chronic low back pain: Concepts and hypotheses on contributing mechanisms. Eur J Pain 2003;7:9-21.

8. Jowett RL, Fidler MW, Troup JD. Histochemical changes in the multifidus in mechanical derangements of the spine. Orthop Clin North Am 1975;6:145-61. 


\section{Caby et al}

9. Nachemson A, Lindh M. Measurement of abdominal and back muscle strength with and without low back pain. Scand J Rehabil Med 1969;1:60-3.

10. Polatin PB, Kinney RK, Gatchel RJ, Lillo E, Mayer TG. Psychiatric illness and chronic low-back pain. The mind and the spine - which goes first? Spine 1993;18:66-71.

11. Robert J. Gatchel, Tom G. Mayer. Evidence-informed management of chronic low back pain with functional restoration. Spine J 2008;8:65-69.

12. Carragee EJ. Clinical practice. Persistent low back pain. N Engl J Med 2005;352:1891-8.

13. Genet F, Lapeyre E, Schnitzler A, et al. [Psychobehavioural assessment for chronic low back pain]. Ann Readapt Med Phys 2006;49:226-33.

14. Merboth MK, Barnason S. Managing pain: The fifth vital sign. Nurs Clin North Am 2000;35:375-83.

15. Zanoli G, Stromqvist B, Jonsson B. Visual analog scales for interpretation of back and leg pain intensity in patients operated for degenerative lumbar spine disorders. Spine 2001;26:2375-80.

16. Huskisson EC. Measurement of pain. Lancet 1974;2:1127-31.

17. McGeary DD, Mayer TG, Gatchel RJ. High pain ratings predict treatment failure in chronic occupational musculoskeletal disorders. J Bone Joint Surg Am 2006;88:317-25.

18. Mayer TG, Gatchel RJ. Functional restoration for spinal disorders: The sports medicine approach. Philadelphia: Lea \& Febiger, 1988.

19. Lawlis GF, Cuencas R, Selby D, McCoy CE. The development of the Dallas Pain Questionnaire. An assessment of the impact of spinal pain on behavior. Spine 1989;14:511-6.

20. Cresswell AG, Oddsson L, Thorstensson A. The influence of sudden perturbations on trunk muscle activity and intra-abdominal pressure while standing. Exp Brain Res 1994;98:336-41.

21. Gauvin MG, Riddle DL, Rothstein JM. Reliability of clinical measurements of forward bending using the modified fingertip-tofloor method. Phys Ther 1990;70:443-7.

22. Perret C, Poiraudeau S, Fermanian J, Colau MM, Benhamou MA, Revel M. Validity, reliability, and responsiveness of the fingertip-tofloor test. Arch Phys Med Rehabil 2001;82:1566-70.

23. Liemohn W, Sharpe GL, Wasserman JF. Criterion related validity of the sit-and-reach test. J Strength Cond Res 1994;8:91-94.

24. Biering-Sorensen F. Physical measurements as risk indicators for low-back trouble over a one-year period. Spine 1984;9:106-19.

25. Keel PJ, Wittig R, Deutschmann R, et al. Effectiveness of in-patient rehabilitation for sub-chronic and chronic low back pain by an integrative group treatment program (Swiss Multicentre Study). Scand J Rehabil Med 1998;30:211-9.

26. Mayer TG, Barnes D, Nichols G, et al. Progressive isoinertial lifting evaluation. II. A comparison with isokinetic lifting in a disabled chronic low-back pain industrial population. Spine 1988;13:998-1002.
27. Institut National de Recherche et de Sécurité. Lombalgies et travail : pour une stratégie consensuelle. Documents pour le médecin du travail, 2ème trimestre. Paris: INRS, 2002.

28. Garzillo MJ, Garzillo TA. Does obesity cause low back pain? J Manipulative Physiol Ther 1994;17:601-4.

29. Smeets RJ, Vlaeyen JW, Hidding A, et al. Active rehabilitation for chronic low back pain: Cognitive-behavioral, physical, or both? First direct post-treatment results from a randomized controlled trial. BMC Musculoskelet Disord 2006;7:5.

30. Wright LJ, Schur E, Noonan C, Ahumada S, Buchwald D, Afari N. Chronic pain, overweight, and obesity: Findings from a communitybased twin registry. J Pain 2010;11:628-35.

31. Nguyen C, Poiraudeau M, Revel M, Papelard A. Lombalgie chronique : facteurs de passage à la chronicité. Revue du Rhumatisme 2009;76:537-42.

32. Shirado O, Ito T, Kikumoto T, Takeda N, Minami A, Strax TE. A novel back school using a multidisciplinary team approach featuring quantitative functional evaluation and therapeutic exercises for patients with chronic low back pain: The Japanese experience in the general setting. Spine 2005;30:1219-25.

33. Thomas E, Olive P, Canovas F, et al. Tophaceous gout of the navicular bone as a cause of medial inflammatory tumor of the foot. Foot Ankle Int 1998;19:48-51.

34. Demoulin C, Crielaard JM, Vanderthommen M. Spinal muscle evaluation in healthy individuals and low-back-pain patients: A literature review. Joint Bone Spine 2007;74:9-13.

35. van Weering MG, Vollenbroek-Hutten MM, Tonis TM, Hermens HJ. Daily physical activities in chronic lower back pain patients assessed with accelerometry. Eur J Pain 2009;13:649-54.

36. Vlaeyen JW, de Jong J, Geilen M, Heuts PH, van Breukelen G. The treatment of fear of movement/(re)injury in chronic low back pain: Further evidence on the effectiveness of exposure in vivo. Clin J Pain 2002;18:251-61.

37. Hietanen E. Cardiovascular responses to static exercise. Scand J Work Environ Health 1984;10(6 Spec No):397-402.

38. Masuda K, Masuda T, Sadoyama T, Inaki M, Katsuta S. Changes in surface EMG parameters during static and dynamic fatiguing contractions. J Electromyogr Kinesiol 1999;9:39-46.

39. Fisher JP, White MJ. Muscle afferent contributions to the cardiovascular response to isometric exercise. Exp Physiol 2004;89:639-46.

40. Bontoux L, Dubus V, Roquelaure Y, et al. Return to work of 87 severely impaired low back pain patients two years after a program of intensive functional rehabilitation. Ann Phys Rehabil Med 2009;52:17-29.

41. Al-Obaidi SM, Nelson RM, Al-Awadhi S, Al-Shuwaie N. The role of anticipation and fear of pain in the persistence of avoidance behavior in patients with chronic low back pain. Spine 2000;25:1126-31. 


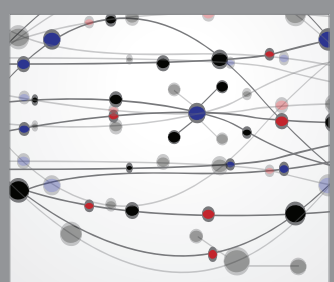

The Scientific World Journal
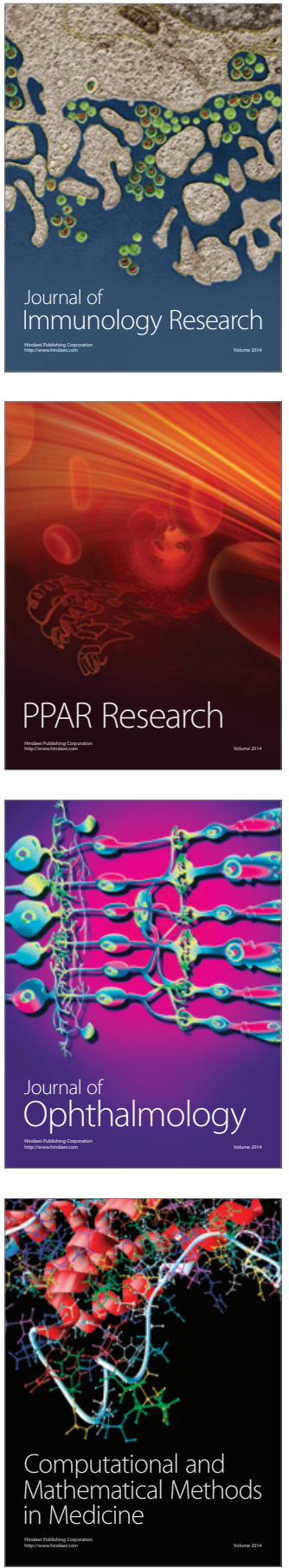

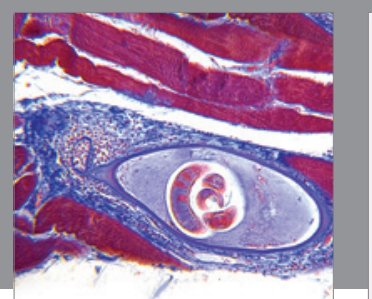

Gastroenterology Research and Practice

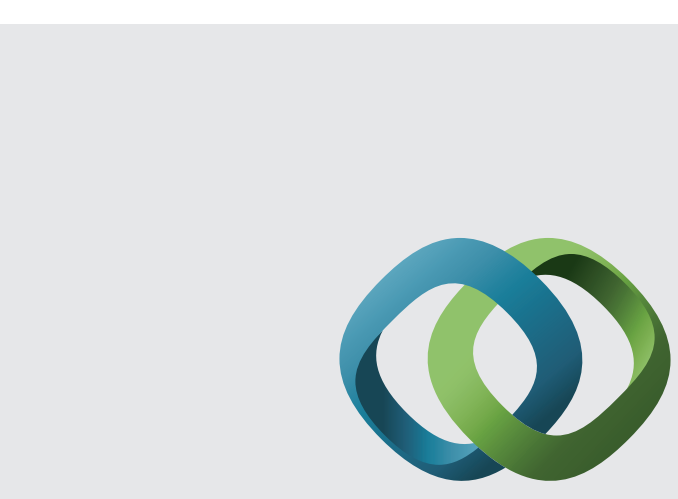

\section{Hindawi}

Submit your manuscripts at

http://www.hindawi.com
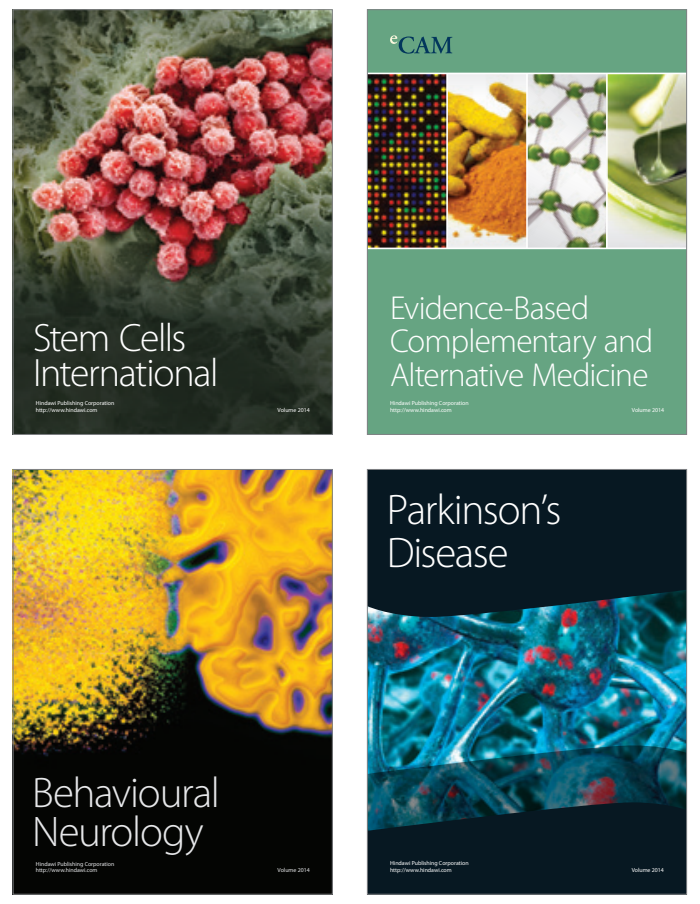
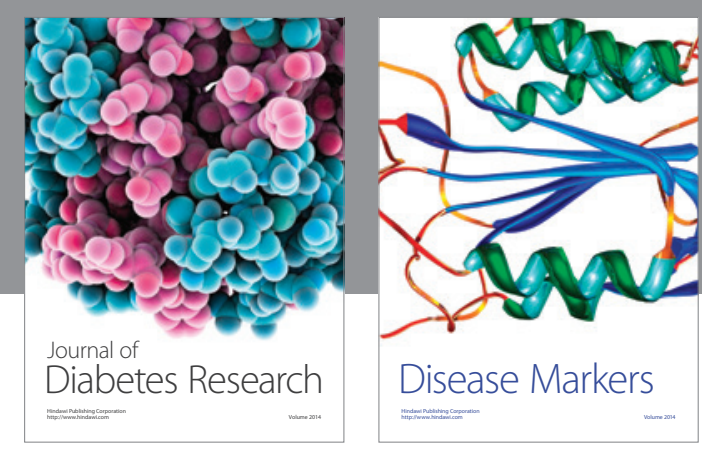

Disease Markers
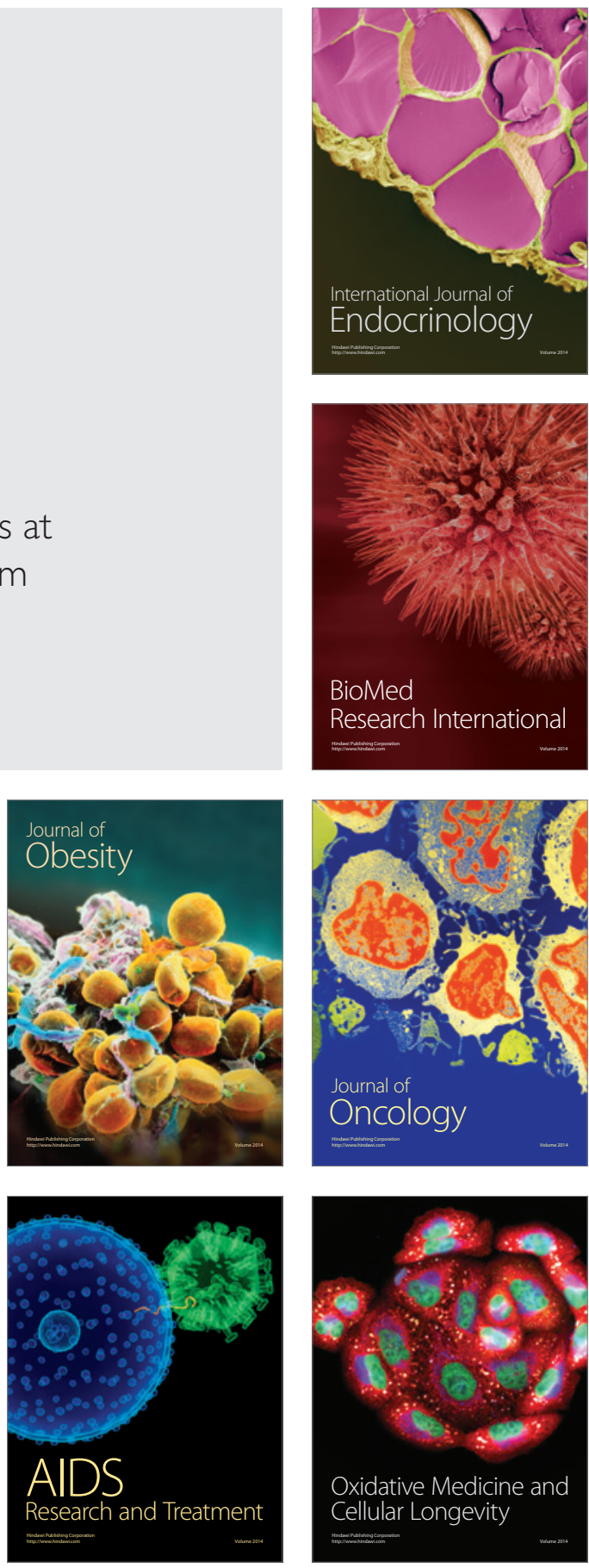